\title{
The Influence of the Lewis Number on the Propagation of a Plane Thermal Wave
}

\author{
V. Frost \\ Ishlinsky Institute for Problems in Mechanics, RAS, \\ Moscow, 119526, Russia \\ frost@ipmnet.ru
}

\begin{abstract}
The results of a numerical calculating of the propagation of plane wave of reaction described by the equations of one-dimensional unsteady heat conduction and diffusion are presented. To describe the rates of energy supply and the change in mixture composition, macrokinetic equations are used. The set of dimensionless variables used is such that, in the simplest case (when thermal parameters and density are constant), only the igniter temperature, the initial temperature of the mixture and the ratio of activation energy to calorific value of a fuel, have a decisive influence on the propagation process. The reaction rate constant enters only in the recount of results into physical variables and does not affect the stability limits. In the range of Lewis numbers from 0 to 1 , stationary, oscillatory, and pulsating modes of reaction wave propagation are obtained. The boundaries that determine the types of the propagating thermal waves are constructed.
\end{abstract}

Keywords: Lewis number, flame instability, pulsating flames

doi: $10.33257 / \mathrm{PhChGD} \cdot 20.1 .764$

The results of a numerical calculation of the propagation of a plane reaction wave described by the equations of thermal conductivity and diffusion, containing expressions for the rates of energy supply and changes in the composition of the mixture, are presented. All thermophysical parameters are assumed to be constant. The used dimensionless variables such, that the determining variables are only the Lewis number, the dimensionless initial temperature and the complex $\mathrm{E}=E /\left(R T_{F}\right)$ containing the activation energy of the reaction and the thermal efficiency of the fuel. Various modes of wave forming were obtained: 1) occurrence of propagation with a constant rate of combustion which is determined by the rate of the active component decreasing, 2) the occurrence of decreasing and 3) non-decreasing fluctuations in the rate of combustion and 4) regimes in which the propagation velocity varies from very high values in very short periods to very small values in relatively long periods. These modes can be called pulsating. The figure shows the boundaries between modes 1 and 2 and 2 and 3. Modes 4 are located below modes 3 .

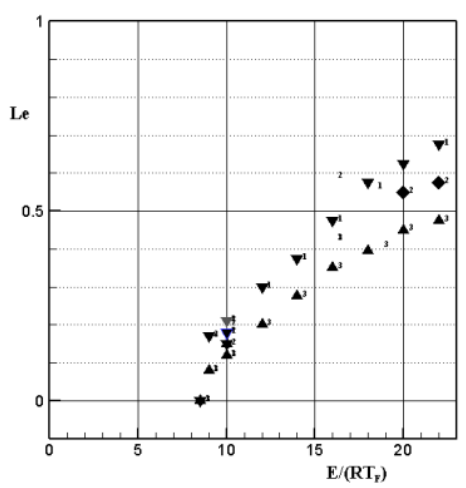

Fig. Below $\boldsymbol{\Delta}$ are modes of sustained oscillations of the rate of combustion and pulsating modes, above there is a steady stationary propagation, between these signs there is a region of damped oscillations 
УДК 544.427

\title{
Влияние числа Льюиса на распространение плоской тепловой волны
}

\author{
В.A. Фрост \\ Институт проблем механики им. А.Ю. Иилинского РАН, \\ Россия, Москва, 119526, пр-т Вернадского, д. 101, корп. 1 \\ frost@ipmnet.ru
}

\begin{abstract}
Аннотация
Приведены результаты численного расчета распространения плоской волны реакции, описываемого одномерными нестационарными уравнениями теплопроводности и диффузии. Для описания скоростей подвода энергии и изменения состава смеси используются макрокинетические уравнения. Используемый набор безразмерных переменных, таков, что определяющее влияние на процесс распространения в простейшем случае постоянства теплофизических параметров и плотности оказывают температура воспламенителя, начальная температура смеси и отношение энергии активации к теплотворной способности горючего. Константа скорости реакции входит только в коэффициенты пересчета результатов в физические переменные и не влияет на границы устойчивости. В диапазоне чисел Льюиса $\mathrm{Le}=D / \kappa$ от 0 до 1 получены стационарные и пульсирующие режимы распространения волны реакции.
\end{abstract}

Ключевые слова: число Льюиса, неустойчивость пламени, пульсирующие пламена.

doi: $10.33257 / \mathrm{PhChGD} \cdot 20.1 .764$

\section{1. Введение}

Приведены результаты численного расчета распространения плоской волны реакции, описываемого одномерными нестационарными уравнениями теплопроводности и диффузии, содержащими выражения для скоростей подвода энергии и изменения состава смеси. Целью исследования является изучение влияния числа Льюиса $(\mathrm{Le}=D / \kappa)$ на особенности процесс распространения плоской волны реакции, а также определение границ устойчивости стационарного распространения плоской волны реакции.

Такие исследования имеют довольно длительную историю, начало которой положили работы 1938 г. Я.Б. Зельдовича и Д.А. Франк-Каменецкого [1, 2], в которой, используя подобие профилей температуры и концентрации, существующее при числе Льюиса равном единице, удалось построить асимптотику для бесконечного значения энергии активации и создать метод расчета скорости стационарного распространения пламени, что составило основу теории распространения пламени.

Проблема связи числа Льюиса и устойчивости пламени возникла несколько ранее. В работе 1934 г. [3] Б. Льюис и Г. Эльбе обнаружили, что при коэффициенте температуропроводности $\kappa$, большем коэффициента диффузии $D$, в волне реакции существует максимум полной энтальпии, т. е. возникает состояние, в котором, как правило, система оказывается неустойчивой. В дальнейшем вопрос об устойчивости пламени чаще всего рассматривали как возникновение пространственной структуры в результате потери устойчивости плоского пламени. Значительное влияние на дальнейшие исследования в этом направлении оказала работа Л.Д. Ландау 1944 г. [4], в которой была показана абсолютная неустойчивость бесконечно тонкого фронта пламени по отношению к возмущениям движения среды. При наличии 
возмущений среды возникают растущие с течением времени возмущения первоначально плоского разрыва между холодной горючей смесью и продуктами сгорания.

При проведении численных расчетов необходима уверенность в существовании искомого объекта. В рассматриваемом случае таким объектом является волна реакции, распространяющаяся по среде без изменения своей формы, то есть ищется стационарное решение. Условия существования и единственности стационарного решения диффузионных уравнений, как было показано в работе А.Н. Колмогорова, Г.И. Петровского и Н.С. Пискунова 1937 г. [5], зависят от свойств функции, описывающей скорость превращения переносимого компонента. Доказательство существования стационарного решения задачи о распространении пламени для химической реакции первого порядка при произвольном постоянном числе Льюиса и $\kappa \geq D$ приведено в книге [6] (стр. 209). В дальнейшем С.С. Новиков и Ю.С. Рязанцев [7] обобщили доказательство этого факта на случай переменного зависящего от температуры числа Льюиса и доказали единственность решения. В работах [8-10] показано, что в случае $\kappa<D$ возможна неединственность решения.

Из работ по доказательствам существования и единственности решения стационарной задачи о распространении следует практический вывод: численное решение задачи о распространении пламени надо строить, положив скорость реакции равной нулю в некотором интервале значений температуры, включающим в себя начальную температуру [6]. В производимых расчетах предполагается, что реакция отсутствует при температурах меньших одной тысячной от максимально возможного значения.

Большинство численных исследований распространения волны реакции выполнено применительно к задачам самораспространяющегося сверхтемпературного синтеза (CBC), в условиях которого диффузия отсутствует, число Льюиса равно нулю, исходные вещества и продукты реакции все время находятся в твёрдой фазе. Достаточно полное представление о процессах, происходящих при Le $=0$ дают исследования [11], посвященные возникновению пульсирующих режимов горения.

Инициирование горения, как правило, производится заданием начального профиля волны или контактом с нагретой поверхностью. В последнем случае начальную стадию процесса можно рассматривать как последствие достаточно сильного воздействия, а развивающийся процесс как результат жесткого возбуждения.

К моменту начала наших исследований теоретическая база и вычислительная практика были достаточно развиты, однако, исследований влияния числа Льюиса в широком диапазоне его изменений в научной литературе обнаружено не было. Только в работе [12] приведены результаты для нескольких значений числа Льюиса, подтверждающие теоретические выводы. Исследование влияния числа Льюиса в диапазоне $(0,1)$ представлено в данной работе.

Расчеты проводятся в максимально упрощенных условиях, что позволяет четко выделить основные детали процесса формирования волн реакции. Все теплофизические параметры предполагаются постоянными. Постоянство плотности позволяет не рассматривать движение среды. Используются безразмерные переменные, в результате определяющими переменными являются число Льюиса, безразмерная начальная температура и комплекс $\mathrm{E}=E /\left(R T_{F}\right)$, содержащий энергию активации реакции и тепловую эффективность топлива $T_{F}$. В качестве характеристики скорости распространения волны используется скорость сгорания, определяемая по скорости убыли активного компонента. Употребление для этого скорости перемещения характерной точки волны, задаваемой выбранным значением некоторого параметра, неудобно, так как в условии быстрого изменения скорости сгорания положение выбранной точки зависит не только от скорости перемещения волны, но и от перестройки ее профиля.

Для расчета используется метод прогонки [12], количество расчетных точек примерно 1000 на единицу длины, шаг по времени выбирался из условий отсутствия его влияния на конечные результаты, зона реакции распространяется по полубесконечной пространственной оси, в расчетах длина пространственного интервала превышала более чем на $25 \%$ расстоя- 
ние, на котором могла оказаться волна горения, на воспламеняющей поверхности используются условия Дирихле для температуры и Неймана для концентрации активного компонента. В некоторых случаях у этой поверхности в начальный момент располагается слой продуктов реакции, что в ряде случаев смягчает процесс формирования волны.

Отладка метода расчета проводится при числе Льюиса равном единице, режиме достаточно хорошо изученном.

В процессе расчетов получены различные режимы распространения волны: 1) режимы с постоянной скоростью распространения; 2) режимы с убывающими и неубывающими пульсациями скорости распространения; 3) режимы, в которых скорость меняется от очень высоких значений в течение непродолжительных периодов до небольших значений в течение относительно продолжительных периодов. Эти режимы можно назвать неравномерно пульсирующими.

\section{2. Постановка задачи}

\section{1. Определяющие уравнения}

Рассматриваемый случай является примером термодиффузионного распространения волны реакции, для его описания используются уравнение распространения тепла

$$
\rho\left(x^{0}, t^{0}\right) \frac{\partial c_{p} T^{0}}{\partial t^{0}}=\frac{\partial}{\partial x^{0}} \lambda \frac{\partial T^{0}}{\partial x^{0}}+Q W\left(C^{0}, T^{0}\right)
$$

и уравнение переноса отдельных химических компонентов (под концентрациями понимаются безразмерные относительные парциальные плотности) [6]

$$
\rho\left(x^{0}, t^{0}\right) \frac{\partial C_{i}^{0}}{\partial t^{0}}=\frac{\partial}{\partial x^{0}} \rho\left(x^{0}, t^{0}\right) D^{0} \frac{\partial C_{i}^{0}}{\partial x^{0}}+v_{i} W_{i}\left(C^{0}, T^{0}\right)
$$

Здесь и далее верхний индекс «0» используется для обозначения величин в физических переменных, их безразмерные аналоги обозначаются без верхнего индекса; $\lambda$ - коэффициент теплопроводности; $D^{0}$ - коэффициент диффузии; $T^{0}-$ температура; $C^{0}=\left(C_{1}^{0}, \ldots C_{k}^{0}\right)-$ вектор, компонентами которого являются концентрации рассматриваемых компонентов; $\rho$ - плотность; $c_{p}$ - теплоемкость; $t^{0}$ - время; $x^{0}$ - пространственная координата; $W$ и $W_{i}-$ скорости тепловыделения и изменения концентрации $i$-го компонента в результате реакции; $v_{i}$ - стехиометрический коэффициент соответствующей реакции. Используются простейшие формы выражений скоростей реакции

$$
\begin{aligned}
& W\left(C^{0}, T^{0}\right)=Q k_{r}\left[\rho\left(x^{0}, t^{0}\right) C^{0}\right]^{n} \exp \left(-\frac{E^{0}}{R T^{0}}\right), \\
& W_{i}\left(C^{0}, T^{0}\right)=k_{r i}\left[\rho\left(x^{0}, t^{0}\right) C^{0}\right]^{n} \exp \left(-\frac{E_{i}^{0}}{R T^{0}}\right),
\end{aligned}
$$

где $Q$ - тепловой эффект реакции; $k_{r}$ - константы скоростей реакции; $n$ - порядок реакции.

Рассматривается простейший случай одного активного компонента, сводящий систему уравнений к двум уравнениям. В этом случае предполагая параметры $\rho^{0}=\rho\left(x^{0}, t^{0}\right)$, $\kappa=\frac{\lambda}{\rho^{0} c_{p}}$ и, вводя число Льюиса $\mathrm{Le}=D / \kappa$, переходим к безразмерным переменным 


$$
\begin{gathered}
\frac{\partial T}{\partial t}=\frac{\partial}{\partial x} \frac{\partial T}{\partial x}+W(C, T), \\
\frac{\partial C}{\partial t}=\operatorname{Le} \frac{\partial}{\partial x} \frac{\partial C}{\partial x}+W(C, T)
\end{gathered}
$$

При этом в определении безразмерных параметров используется интеграл

$$
W_{I}=\int f\left(T^{0}\right) \exp \left(-\frac{E^{0}}{R T_{F}^{0}}\right) \mathrm{d} T^{0},
$$

при вычислении которого используется предположение о подобии полей температуры и концентрации, справедливое для установившегося распространения волны реакции при числе Льюиса равном единице. Интегрирование проводится по области, в которой возможна реакция. Интеграл $W_{I}$ является существенной частью закономерности для скорости распространения в теории Д. Франк-Каменецкого и Я. Зельдовича и позволяет скомпенсировать отличие в порядке величин экспоненциального множителя и константы скорости реакции.

В уравнениях (1) и (2) используются следующие безразмерные параметры:

$$
\begin{gathered}
T=\frac{T^{0}-T_{0}^{0}}{T_{F}^{0}-T_{0}^{0}}, T_{F}^{0}=\frac{Q}{\rho c_{p}}, T_{0}=\frac{T_{0}^{0}}{T_{F}^{0}}, \mathrm{E}=\frac{E^{0}}{R T_{F}^{0}}, \quad t=\frac{t^{0}}{Q k_{r} W_{I}}, x=\frac{x^{0}}{\sqrt{\left(\kappa Q k_{r} W_{I}\right)}}, \\
W(C, T)=\frac{C^{n} \exp \left[-\frac{\mathrm{E}}{R\left(T_{0}+T T_{F}^{0}\right)}\right]}{W_{I}}
\end{gathered}
$$

\section{2. Граничные условия, инициация процесса распространения}

Рассматривается развитие процесса на пространственной оси $x \subset(0, \infty)$, начало формирования волны происходит около точки $x=0$. Распространение волны происходит в сторону возрастания $x$. Как правило [12] формирование волны инициируется заданием "ступеньки" температуры и концентрации. Однако, если предположить, что начальные условия являются сильным воздействием на последующее развитие неустойчивости, приводящей к возникновению сложных нестационарных процессов, то в качестве инициатора процесса удобно рассматривать нагретую стенку, контактирующую с изначально не реагирующей средой. В рассматриваемом одномерном случае это означает использование условия Дирихле для температуры. Стенка предполагается непроницаемой для активного компонента, поэтому для концентрации используется условие Неймана.

Таким образом

$$
x=0 ; T(0, t)=T_{b}, \frac{\partial C}{\partial x}=0
$$

Здесь $T_{b}$, безразмерная температура воспламенителя, в большинстве расчетов использовалось $T_{b}^{0}=T_{F}^{0}$.

\section{3. Результаты расчетов}

Отладка программы расчета и иллюстрация ее возможностей производились на хорошо изученном случае числа Льюиса равном единице. Существующее в этом случае подобие полей температуры и концентрации выполняется только после удаления волны реакции от точ- 
ки $x=0$. Поэтому производиться решение методом прогонки задачи Коши системы уравнений (1) и (2). Правая граница интервала $x$ располагалась на расстоянии недостижимом для волны за все время расчета.

3.1. Влияние безразмерной энергии активации $\mathrm{E}=\frac{E^{0}}{R T_{F}^{0}}$

Зависимость скорости горения $U n$, определяемой как $U n=-\frac{\mathrm{d}}{\mathrm{d} t} \int_{0}^{\infty} C(x, t) \mathrm{d} x$, от безразмерного параметра $\mathrm{E}=E^{0} /\left(R T_{F}^{0}\right)$ позволяет проверить известное стремление к асимптотическому значению скорости распространения при стремлении этого параметра к бесконечности. Для установившихся режимов, при которых не происходит изменение формы волны, скорость горения совпадает со скоростью распространения любой точки профиля волны. Определяемая подобным образом величина скорости горения характеризует увеличение суммарного количества продуктов горения не зависимо от наличия колебаний и флюктуаций в пространственном случае. На рис. 1. приведены установившиеся на большом расстоянии от воспламенителя в условиях, когда закончился процесс формирования волны, соответствующие зависимости для реакции первого порядка, полученные для начальных температур $T_{0}^{0}=250^{0} \mathrm{~K}, 300^{0} \mathrm{~K}, 350^{0} \mathrm{~K}$. Следует отметить слабое влияние начальной температуры, что может измениться при переходе к физическим переменным. В использующейся системе безразмерных параметров предсказываемая теорией $[1,2]$ скорость распространения равна $\sqrt{2}$, приведенные на рис. 1. результаты не противоречат теории.

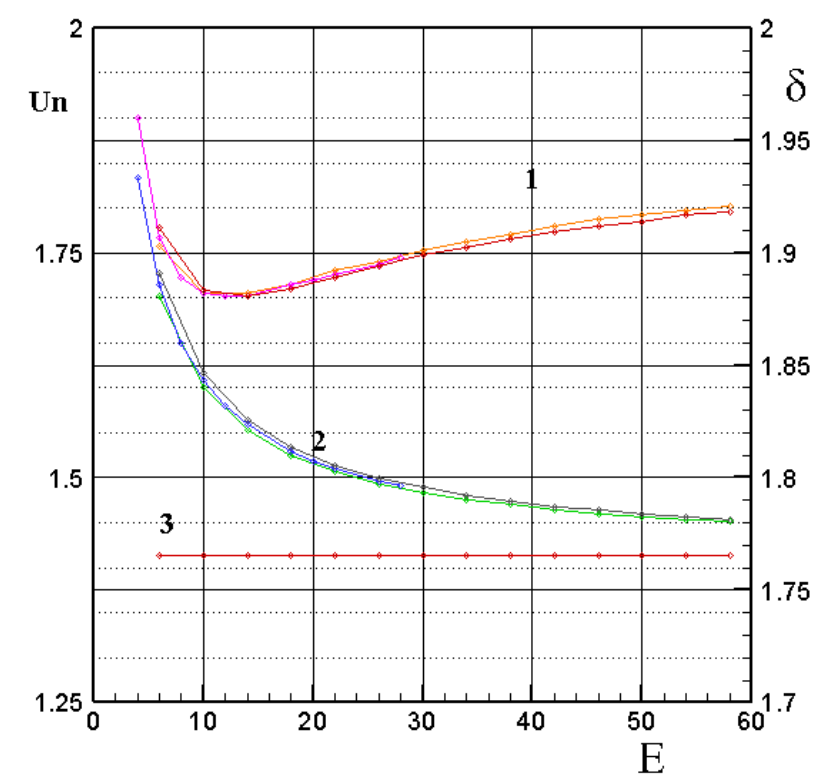

Рис. 1. Характеристики стационарной волны реакции при $\mathrm{Le}=1$ : 1 - толщина фронта $\delta$, определяемая по значениям температуры 0.05 и $0.95 ; 2$ - скорость горения; 3 - величина скорости распространения, предсказываемая теорией [1,2]

На рис. 2. приведены аналогичные результаты для второго порядка реакции. В этом случае стремление к предельной скорости горения значительно более слабое, а также заметно расслоение по начальной температуре. 
Ниже рассматриваются особенности процесса формирования волны, связанные с температурой воспламенителя и использованием прослойки продуктов горения около воспламенителя.

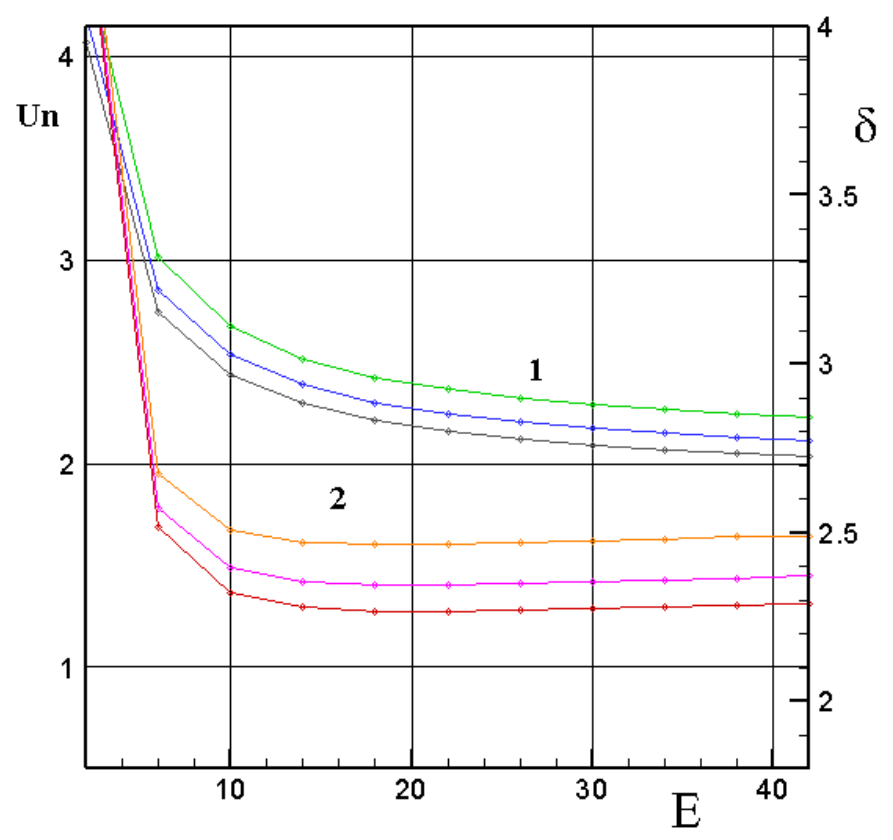

Рис. 2. Аналогично рис. 1. для второго порядка реакции. 1 - скорость горения; 2 - толщина фронта

\section{2. Процесс формирования волны}

Процесс формирования волны изучался при контакте с поверхностью заданной температуры $T_{b}$, которая в основном совпадала с температурой установившегося пламени $T_{F}$, и второй способ, в котором около поверхности воспламенителя располагался слой полностью прореагировавшего состава. Начальные значения в интервале $x \subset(0,1)$ задавались:для температуры равными единице, а для концентрации равными нулю. Характер выхода на стационарный режим для этих способов воспламенения существенно отличался. На рис. 3 приведены соответствующие зависимости от времени скоростей горения.

Расположенная выше кривая соответствует первому способу. В этом случае на начальном участке наблюдается сильное увеличение скорости горения. Во втором случае присутствие слоя продуктов горения у поверхности воспламенителя смягчает процесс формирования стационарного горения. При больших временах оба режима дают практически одинаковые значения скорости горения.

\section{3. Влияние температуры воспламенителя}

Исследование влияния температуры воспламенителя проводилось при первом способе организации формирования стационарного режима. В основном использовалась температура воспламенителя $T_{b}$ равная температуре продуктов горения при установившейся скорости распространения волны горения (температуры пламени). Уменьшение этой температуры приводит к увеличению времени формирования волны и увеличению расстояния от воспламенителя, на котором это формирование происходит. Даже при очень сильном уменьшении температуры воспламенителя воспламенение происходит всегда, так как в рассматриваемом случае отсутствуют потери тепла. При этом формирование волны происходит на расстояниях в несколько сот раз большем, чем при температуре воспламенителя равной температуре пла- 
мени. Этот результат вряд ли может быть перенесен на реальные условия, в которых пространственное протекание процесса, тепловые потери и различные конвективные движения приводят к существованию предельной температуры воспламенения. На рис. 4 в полулогарифмических координатах приведены зависимости скоростей горения от времени для различных значений температуры $T_{b}$.

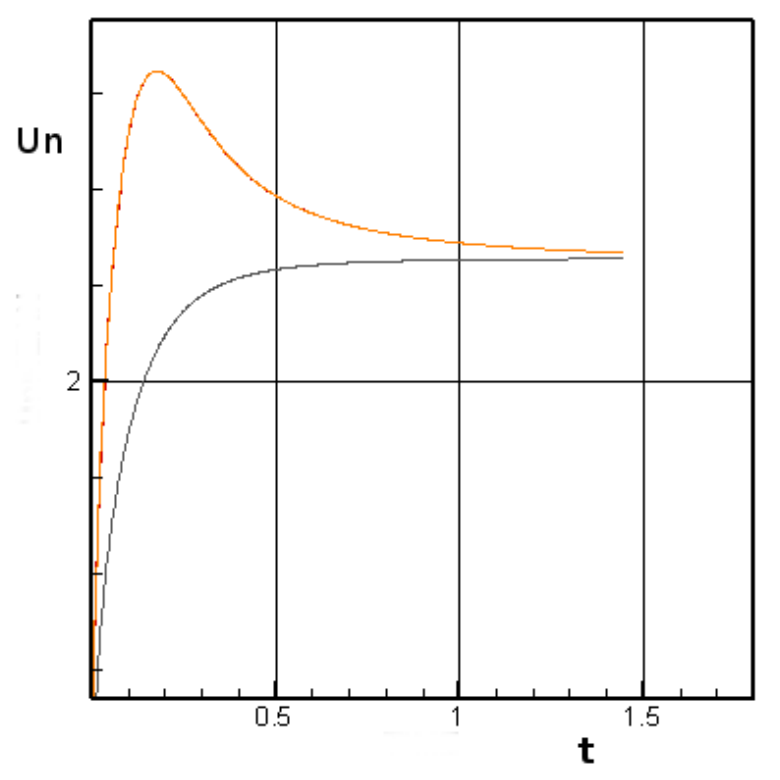

Рис. 3. Два режима формирования стационарной волны реакции. Верхняя кривая - непосредственный контакт с нагретой стенкой, нижняя - наличие слоя толщиной в одну безразмерную единицу сгоревшего вещества у поверхности воспламенителя

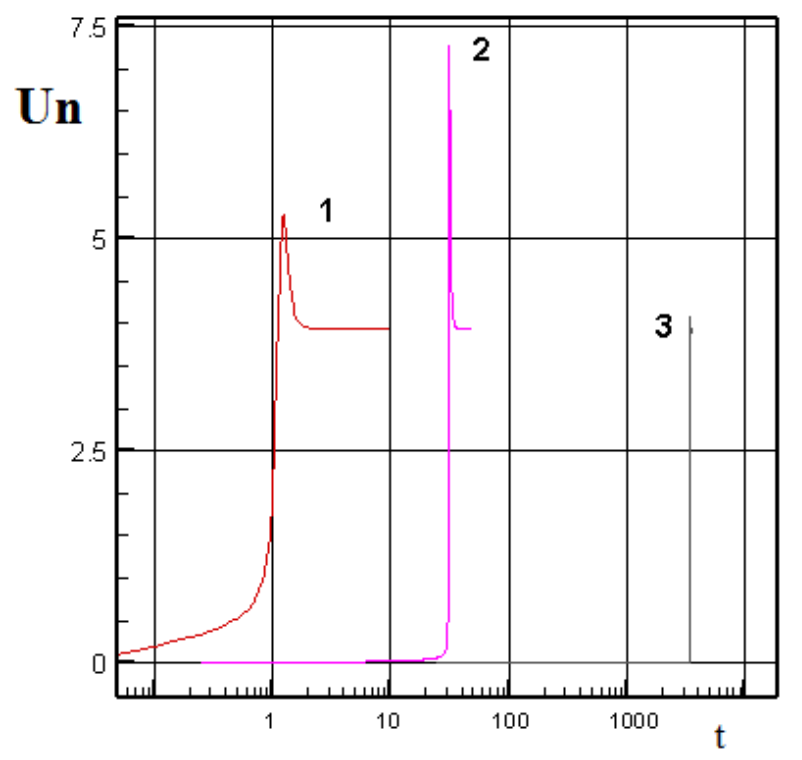

Рис. 4. Режимы формирования стационарной волны горения при различных температурах воспламенителя. $1-1250^{0} \mathrm{~K}, 2-625^{0} \mathrm{~K}, 3-312.5^{0} \mathrm{~K}$. Последняя кривая обозначает только момент начала движения волны

\section{4. Волны горения при отличающимся от единицы числе Льюиса}

Проведенные в диапазоне Le $\subset(0,1)$ расчеты позволили получить несколько типичных сценариев формирования волны реакции. Плавный выход на равномерное распространение с постоянной скоростью горения наблюдается при значениях Le близких к единице (см. 
рис. 3). Во время выхода на режим равномерного распространения возможен также один или несколько пиков скорости горения на начальном участке формирования режима (рис. 5).

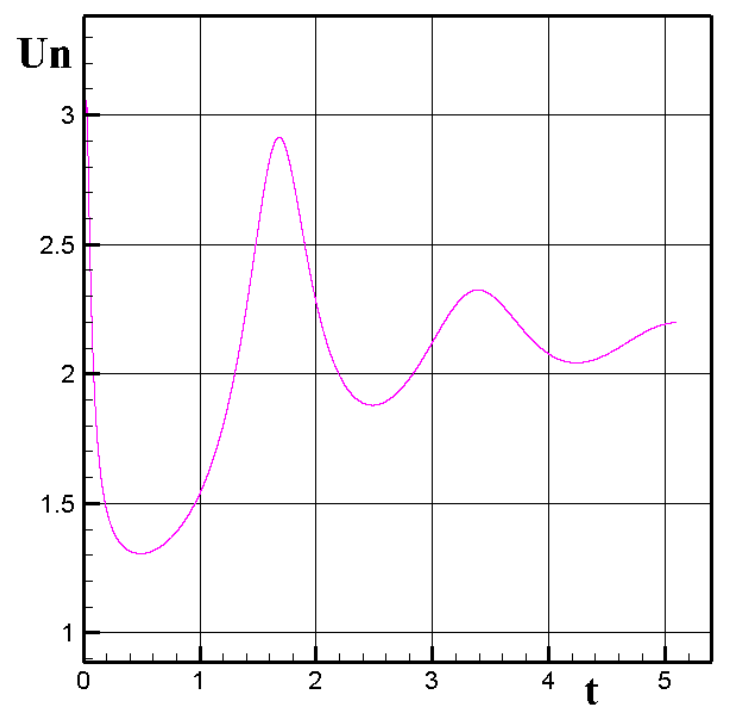

Рис.5. Режим формирования волны реакции вблизи границы перехода от стационарного распространения к колебательному

Эти режимы с несколькими пиками похожи на колебательные режимы с очень большим декрементом. При уменьшении числа Льюиса режимы оказываются ближе к точке потери устойчивости стационарной волны, и наблюдается возникновение колебаний скорости распространения, амплитуда которых с течением времени уменьшается. Уменьшение происходит очень медленно, что позволяет увидеть выход на установившийся режим только при очень слабых колебаниях. При дальнейшем уменьшении числа Льюиса происходит достижение границы устойчивости, и колебания скорости перестают уменьшаться. На рис. 6, $a$ приведены зависимости скоростей распространения для таких режимов. При дальнейшем уменьшении числа Льюиса происходит обострение максимумов на зависимостях для скоростей распространения (рис. 6, б). Средние скорости распространения для этих режимов практически совпадают, но для большей четкости кривые разнесены по вертикали.

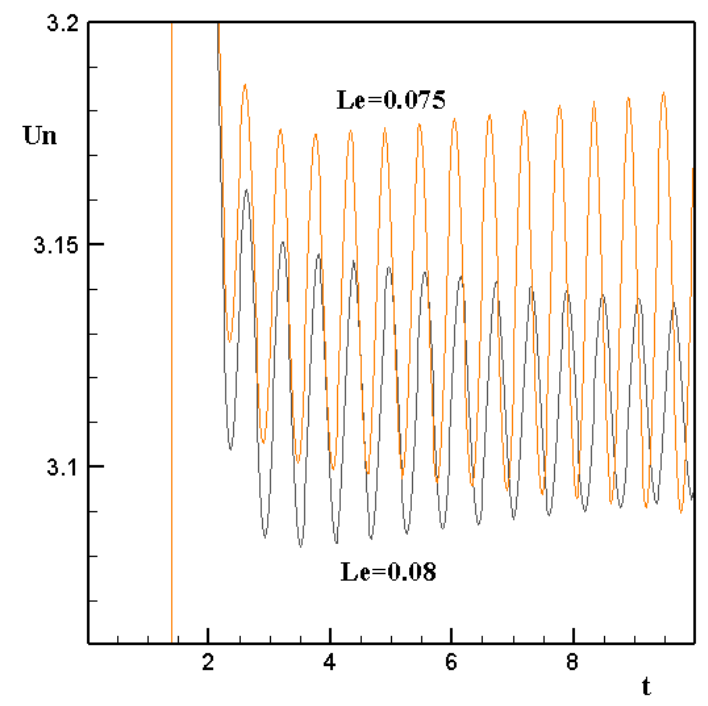

$a$

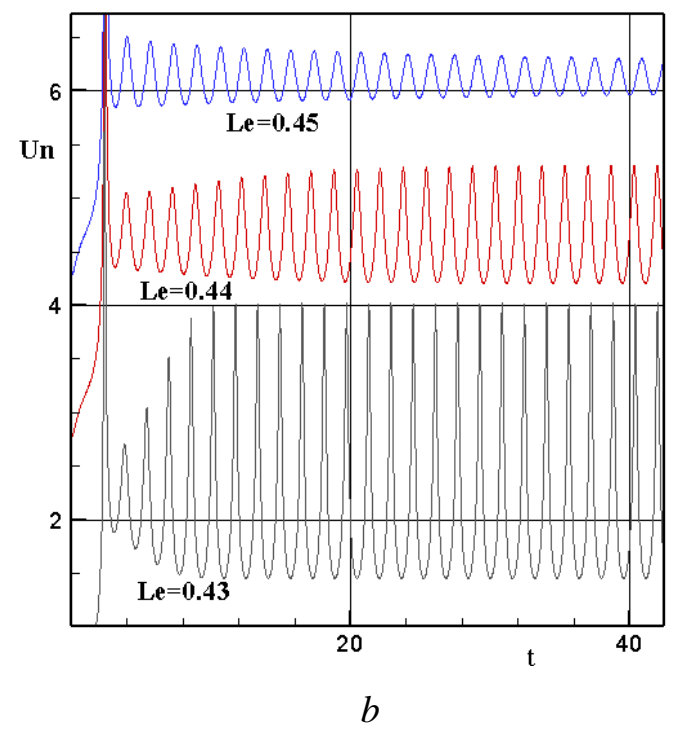

Рис. 6. Сценарии формирования волны горения, для типа 2 и 3 a), различных форм пульсационного распространения волн горения б). На рис. б) для верхних кривых искусственно изменена средняя скорость горения 
С удалением от границы устойчивости происходящее обострение зависимостей для скоростей приводит к возникновению пиков скорости горения (рис. 7). Возникают режимы последовательности очень коротких периодов больших значений скорости распространения чередующихся с относительно продолжительными периодами очень небольших значений. Это так называемые пульсирующие режимы. Наблюдаются режимы с одним или несколькими пиками в каждом из многочисленных повторяющихся циклов. Примеры циклов с одним и двумя пиками в цикле приводится на рис. 6. Возможны и более сложные картины развития процесса.

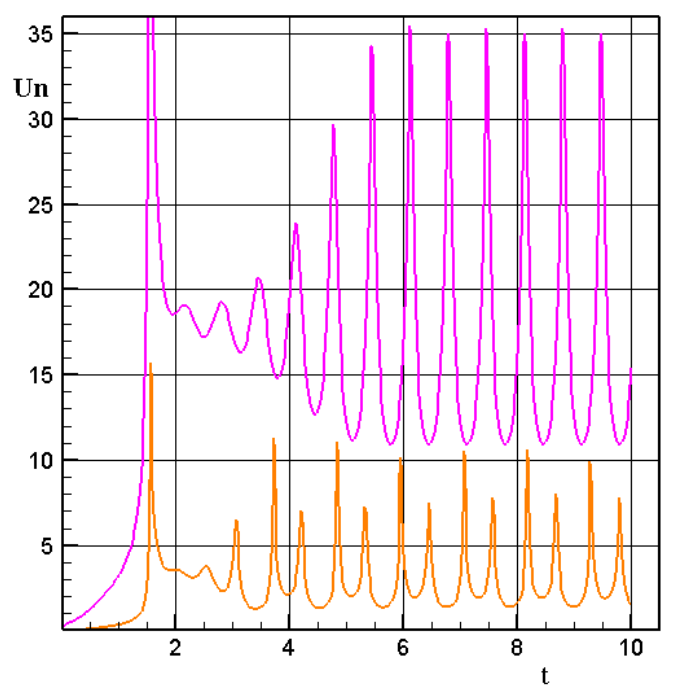

Рис. 7. Одно и двухпиковые режимы зависимостей от времени скорости горения. Кривые за счет искусственного изменения средних скоростей горения искусственно разнесены по вертикали

На рис. 8 изображено изменение скорости горения на некотором участке, довольно далеко расположенном от начала процесса. Изображенную кривую можно было бы трактовать как последовательность из циклов по четыре пика, если бы отсутствовала модуляция максимальных значений. Возможно, это пример режима, который в работе [14] назван хаотическим.

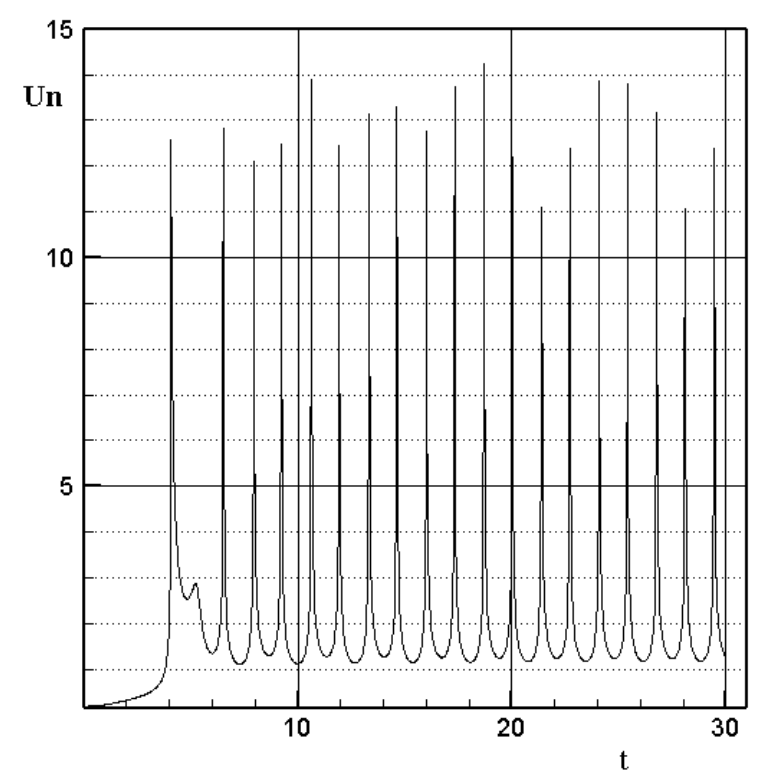

Рис. 8. Формирование многопиковой конфигурации, сопровождаемой низкочастотной модуляцией пиковых значений 
Полученные сценарии формирования волн в основном совпадают с результатами работы [14], в которой для равного единице числа Льюиса численно исследовалось влияние граничного теплового потока на потерю устойчивости плоской волны горения.

Рисунок $6, a$ также иллюстрирует точность определения границ между различными режимами. Переход от затухающих колебаний к незатухающим для данного случая происходит в интервале чисел Льюиса $0.075 \div 0.08$.

В зависимости от характера волны горения изменяются также распределения температуры и концентрации, которые начинают зависеть от фазы цикла. На рис. 9 приведены профили температуры, концентрации (строится отличие концентрации от единицы) и их суммы, которая пропорциональна полной энергии, для характерных моментов времени развития процесса. Рисунок 9, $a$ соответствуют моменту начала распространения волны. В этом случае профили температуры и концентраций похожи на соответствующие профили в стационарно распространяющейся волне горения. Профиль концентрации несколько уже, что естественно для числа Льюиса меньшего единицы. Отличие их суммы от единицы определяется числом Льюиса при значениях больших единицы и участок, на котором эта сумма оказывается меньше единицы, определяется влиянием начального нагрева.

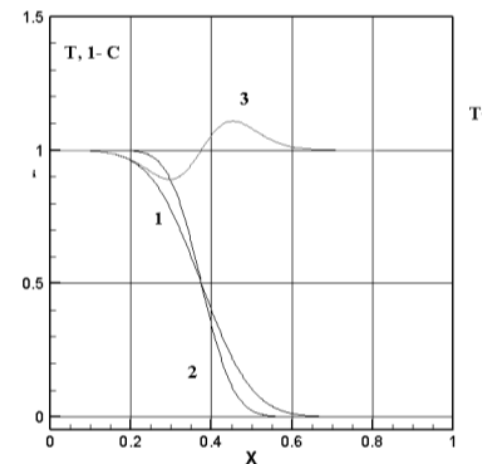

$a$

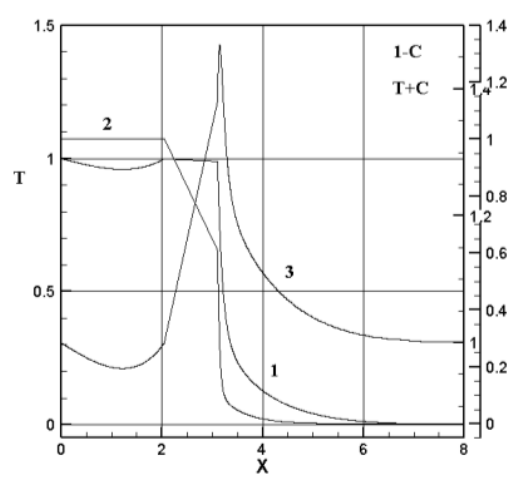

$\sigma$

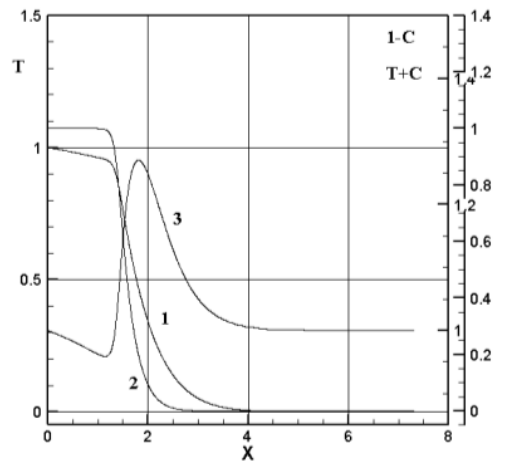

B

Рис. 9. Профили 1 - температуры, 2 - концентрации и 3 - их суммы $a$ ) на начальном участке формирования пульсационного режима; б) в момент первого достижения максимального значения скорости реакции; в) в момент минимальной скорости реакции. Начальной точкой изображения профилей является точка, в которой температура уменьшилась на 0.0001

В момент пикового значения скорости реакции (рис. 9, б) профиль концентрации имеет характерный вид, позволяющий четко разделить зоны подогрева и реакции. В последней наблюдается линейный профиль концентрации. Профиль температуры в этом случае очень узкий. При медленном распространении горения характерной особенности на профилях не наблюдаются (рис. 9, в). В задней части профилей температуры, соответствующих максимальным значениям скорости реакции, наблюдаются характерные провалы, которые с удалением от воспламенителя становятся более длинными, но менее глубокими, что позволяет трактовать их как след от первоначального подогрева на стадии, предваряющей формирование волны. Особенности на профилях концентрации, наблюдаемых в момент пиков, не изменяются с удалением от воспламенителя.

Более наглядным является изображение полей параметров, на рис. 10 приведены поля $T(x, t)$ для режима формирования устойчивого распространения волны горения (рис. $10, a)$ и для режима возникновения пульсирующего режима повторяющихся пиков большой скорости (рис. 10, б), в этом случае изображается поле температуры заданной ширины по вертикали с начальной точкой, в которой температура равна 0.95 . В первом случае после непродолжительного начального периода наблюдается распространение плоской волны с постоянной скоростью распространения. Во втором случае пиковому значению скорости сгорания пред- 
шествует резкое сужение профиля за счет уменьшения зоны реакции. На последующем за пиком спаде скорости горения толщина профиля и зоны реакции увеличиваются.

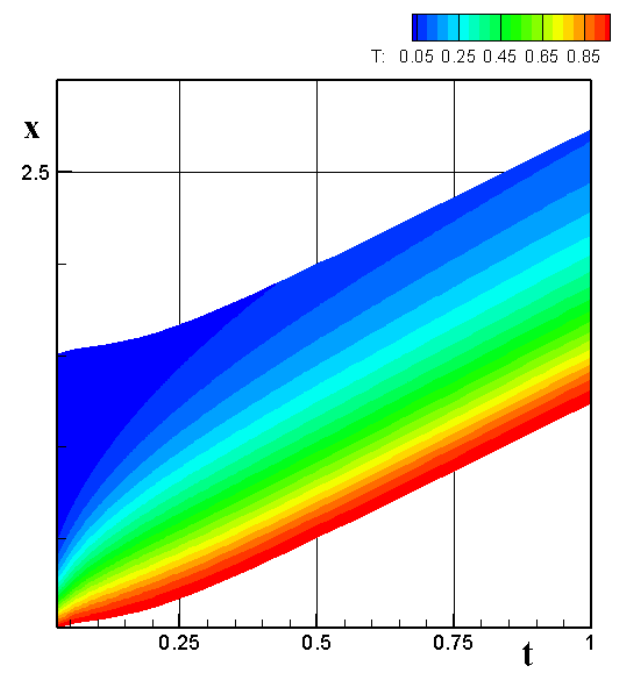

$a$

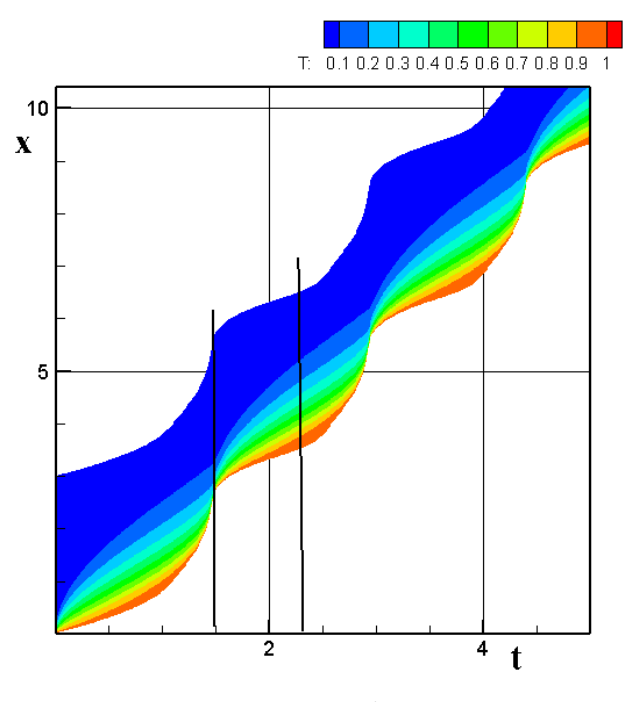

$b$

Рис. 10. Поля $T(x, t)$ для режима возникновения устойчивого распространения волны горения $a$ ) и для режима пульсационного распространения б). Вертикальные прямые обозначают момент первого пикового и минимального значений скорости сгорания

На рис. 11 в координатах ( Le, $\mathrm{E}=E /\left(R T_{F}\right)$ ), расположены точки $\boldsymbol{\nabla}$, соответствующие режимам формирования, близким к переходам от плавного формирования стационарных режимов к режимам, в которых скорость горения испытывает колебания, и точки $\boldsymbol{\Delta}$, соответствующие переходу от последних к незатухающим колебания.

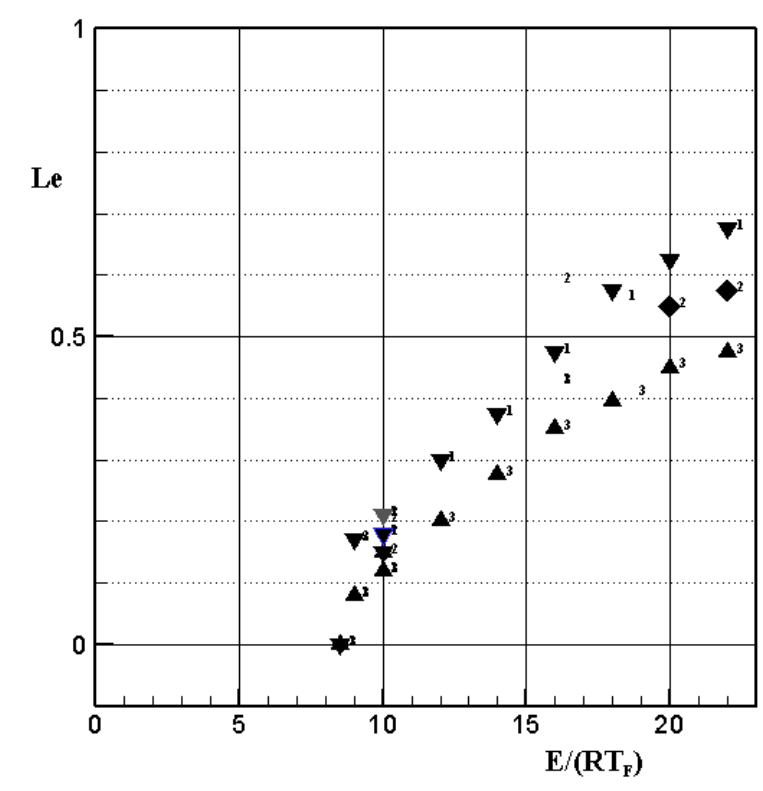

Рис. 11. Расположение в координатах ( Le, $\left.\mathrm{E}=E /\left(R T_{F}\right)\right)$, точек $\boldsymbol{\nabla}$, соответствующих переходу от формирования стационарных режимов к режимам, в которых скорость горения испытывает затухающие колебания, и перехода от них к незатухающим колебаниям: точки $\boldsymbol{\Delta}$

Следует отметить, что определение начального значения числа Льюиса, при котором возникают затухающие колебания, весьма условно и зависит от способа выявления колебаний. Возможно, что колебания малой амплитуды не определяются должным образом. Суще- 
ствование границы перехода от монотонного развития процесса к развитию при наличии затухающих колебаний могут ответить аналитические методы исследования. Граница перехода от затухающих колебаний к незатухающим выделяется достаточно четко. Обнаруживается сближение обеих границ при уменьшении числа Льюиса, так, что при числе Льюиса равном нулю, в области значений параметра $\mathrm{E} \leq 8.5$ находятся режимы выхода на стационарное распространение волн реакции.

\section{4. Заключение}

Проведенные численные расчеты позволили получить границы, разделяющие режимы с устойчивым распространением волны горения с постоянной скоростью и режимы с колебаниями скорости горения. Равенство декремента затухания нулю определяет переход к неустойчивым режимам. С удалением от границы устойчивости наблюдаются режимы с короткими периодами очень высоких скоростей горения, чередующиеся с продолжительными периодами малых скоростей, которые могут быть названы пульсационными. С удалением от границы устойчивости возрастает сложность повторяющихся циклов. Возникающая низкочастотная модуляция не позволяет выделить повторяющиеся фрагменты.

При нулевом числе Льюиса и малых значениях параметра Е обнаружены режимы с постоянной скоростью горения.

Исследование устойчивости стационарного распространения волны горения численными методами является достаточно затратным методом. Основная трудность производимых расчетов связана с очень резкими изменениями скорости горения, что приводит к необходимости считать с очень малыми шагами по времени. Это не позволяет в ряде случаем достичь полного установления. Использование предложенного набора безразмерных переменных не решило проблему малого временного шага. В результате точность положения границ, определяющих переходы между различными типами процессов, определены приближенно. Методы, позволяющие рассчитывать движение медленно меняющихся образований, которые могли бы помочь решить эту проблему, отсутствуют.

Целесообразно проверить полученные результаты аналитическими методами.

\section{Благодарности и ссылки на гранты}

Благодарю В.Н. Маршакова и Э.В. Теодоровича за плодотворные обсуждения.

Работа выполнена по теме государственного задания (№ госрегистрации АAАA-A17117021310385-6).

\section{Литература}

1. Зельдович Н.Б., Франк-Каменецкий Д.А. К теории равномерного распространения пламени // Докл. Акад. Наук. 1938, т. 19. С. 693-698.

2. Зельдович Я.Б., Франк-Каменецкий Д.А. Теория теплового распространения пламени// ЖФХ. 1938.т. 12, вып. 1. С. 100-105.

3. Lewis B., Elbe G. On the theory of flame propagation//J. Chem. Phys. 1934. Vol. 2. N 8, pp. 537-546.

4. Ландау Л.Д. К теории медленного горения//ЖЭТФ. 1944. Т. 14. вып. 6, С.240-245.

5. Колмогоров А.Н., Петровский И.Г., Пискунов Н.С. Исследование уравнения диффузии, соединенной с возрастанием вещества, и его применение к одной биологической проблеме// Бюллетень МГУ. Сер. А. Математика и Механика. 1937.Т. 1. вып. 6, С. 1-26.

6. Зельдович Я.Б., Баренблатт Г.И., Либрович В.Б., Махвиладзе Г.М. Математическая теория горения и взрыва М.: Наука, 1980. 478 с.

7. Новиков С.С., Рязанцев Ю.С. О существовании и единственности решения системы уравнений тепловой теории горения//ПМТФ. 1965. № 4, С. 86-88. 
8. Бачелис Р.Д., Меламед В.Г. О неединственности стационарных решений для системы уравнений теории горения при кусочно-постоянных константе скорости и коэффициентах теплопроводности п диффузии // Докл. Акад. Наук. 1965. Т. 163. № 6, С. 1338-1341.

9. Бачелис Р.Д., Меламед В.Г. О неединственности стационарного решения системы уравнений теории горения//ПММ. 1966. Т. 30. вып. 2, С. 368-374.

10. Бачелис Р.Д., Меламед В.Г. О неединственности стационарного решения для системы уравнений теории горения в случае постоянного отношения коэффициентов теплопроводности и диффузии//ПМТФ. 1968. .№ 1, С. 161-167.

11. Шкадинский К.Г., Хайкин В.И., Мержанов А.Г.Распространение пульсирующего фронта экзотермической реакции в конденсированной фазе//ФГВ. 1971. Т. 7. № 1, С. 19-28.

12. Zeldovich Y.B., Barenblatt G.I. Theory of flame propagation//Combust. and Flame. 1959. Vol. 3(1). Pp. 61-74.

13. Березин И.С., Жидков Н.П. Методы вычислений М.: Физматлит, 1960. Т. 2. 620 с.

14. Беляев А.А., Каганова З.И., Новожилов Б.В., Горение летучих конденсированных систем за границей устойчивости стационарного режима//Физика горения и взрыва. 2004. Т. 40. №4, C. 60-6. 